\title{
Bimanual Coordination Being Efficient Around Two Months After Stroke: A Key Recovery Moment for Starting Bimanual Rehabilitation Protocols?
}

\author{
Metrot Julien ${ }^{*} \quad$ Laffont Isabelle ${ }^{*}{ }^{* \dagger}$ \\ Bonnin Huey-Yune ${ }^{\star}$ VanDokkum Liesjet ${ }^{*}$ Mottet Denis* \\ (*) Movement To Health, Montpellier-1 University, France \\ (*) Physical Medicine and Rehabilitation Unit, Montpellier CHRU, France \\ (†) Grau du Roi Medical Center, Nîmes CHU, France \\ E-mail: julien.metrot@univ-montp1.fr
}

\begin{abstract}
Better understand how bimanual coordination evolves during the first weeks of natural recovery after stroke is needed to address bimanual rehabilitation. 15 stroke patients realized seven kinematic assessments once a week during six weeks and at three months. The grasping task was performed through 3D-movement analysis in three different conditions: unimanual with the non-paretic limb, unimanual with the paretic limb, and bimanual.

Inter-limb coordination became efficient about 6 weeks after stroke, represented by a plateau phase. Bimanual coordination is optimized around this period of recovery, indicating a possible beneficial effect of bimanual rehabilitation. Moreover, inter-limb coordination recorded at movement onset and at movement goal did not evolve over recovery.
\end{abstract}

\section{Introduction}

After rehabilitation, about $80 \%$ of post-stroke patients still suffer upper limb disability, which impairs their daily living activities and often leads to major incapacities. One of the promising techniques in stroke rehabilitation is the bimanual training, using inter-limb coordination to favor motor recovery. In a bimanual task, both upper limbs influence and are dependent on each other to perform the task. This process entails that the non-paretic limb involves the paretic limb and improves its output [1] but also that the paretic limb compels the non-paretic limb to match the affected limb lower capabilities.

Bimanual training has been proven efficient [2]. However, most studies don't take into account patients' characteristics, such as recovery phases and improvements have not been demonstrated in all stroke patients. Hence, the interest of rehabilitation based on bimanual mode is still controversial [1] and few are known about post-stroke natural recovery of bimanual coordination.

The goal of the present study is to observe and characterize the evolution of movement kinematics in stroke patients during the first three months of standard rehabilitation (no specific bimanual rehabilitation added), and with a focus on bimanual coordination. We also address which relevant factors may predict the efficiency of bimanual rehabilitation.

\section{Method}

12 hemiparetic patients $(65.6 \pm 9.7)$ were included, within 30 days after a primary unilateral ischemic/hemorrhagic stroke. Seven kinematic assessments of grasping movements occurred once a week during 6 weeks starting from inclusion (W0) and at a follow-up assessment 3 months after inclusion (W12).

Patients were asked to grasp a $5 \mathrm{~cm}$ ball lying on the table, and to move it to the target place with comfortable speed. The grasping task was performed in three different conditions: unimanual non-paretic limb, unimanual paretic limb and bimanual movement. In the bimanual condition, patients were asked to activate movement simultaneously.

Kinematic assessment relied on a 3D motion recording system (Polhemus). For each hand, we computed the following variables to summarize the kinematic features: movement time (MT), number of hand velocity peaks (NVP), time to peak velocity (TPV), maximal tangential velocity (Vmax), and between-hand delay at movement beginning $(\triangle B E G)$, ending $(\triangle \mathrm{END})$, and at velocity peak $(\Delta \mathrm{TPV})$.

This is an Open Access article distributed under the terms of the Creative Commons Attribution-Noncommercial License 3.0, which permits unrestricted use, distribution, and reproduction in any noncommercial medium, provided the original work is properly cited. 


\section{Results \& Discussion}

For unimanual reaching, we found that kinematics was altered in the non-paretic limb, but evolved towards fluency during recovery: decrease of NVP, TVP and MT, increase of Vmax.

For bimanual reaching, a common recovery profile emerged from the two-ways with repeated measures ANOVA (session; hand), showing a plateau phase after 3 weeks of standard therapy. This result was illustrated by MT, which was globally higher for the paretic upper-limb $(\mathrm{F}(7,77)=3.55, p=.02)$. After three weeks of treatment, the two limbs had similar MT (Figure 1) but also reached the velocity peak simultaneously (see TPV, $\mathrm{F}(7,77)=11.02, p<.01$ on Figure 3$)$. The kinematics of the non-paretic limb matched with those of the paretic limb in bimanual condition. It seems that bimanual coordination started to be efficient around 6 weeks after the onset of the stroke. If our hypothesis is exact, that could indicate a possible beneficial effect of a bimanual rehabilitation around this period. According to our results, this kind of rehabilitation could be started at the end of the second month post-injury.

The same results have been found concerning NPV (Figure 2). We showed a session effect on NPV $(\mathrm{F}(7,77)=8,92, p<.01)$ after a Greenhouse-Geisser correction. Post hoc Scheffé test indicated a statistical difference between W0 and other sessions. Moreover, there was no statistical difference between the two hands in bimanual $(\mathrm{p}=0.50)$ whereas it is the case in unimanual $(p<.01)$. This result confirms the idea that the non-paretic hand tends to hover around with the paretic hand during bimanual movement.

\section{References}

[1] S. McCombe Waller, and J. Whitall. Bilateral arm training: Why and who benefits? NeuroRehabilitation, 23(1), 29-41, 2008.

[2] JH. Cauraugh, N. Lodha, SK. Naik, and JJ. Summers. Bilateral movement training and stroke motor recovery progress: A structured review and meta-analysis, Human Movement Science, 29(5): 853-70, 2010.

[3] S. Riek, JR. Tresilian, M. Mon-Williams, VL. Coppard, and RG. Carson. Bimanual aiming and overt attention: one law for two hands. Experimental Brain Research, 153(1), 59-75, 2003.

[4] CY. Wu, SH. Chou, CL. Chen, MY. Kuo, TW. Lu, and YC. Fu. Kinematic analysis of a functional and sequential bimanual task in patients with left hemiparesis: intra-limb and interlimb coordination. Disability \& Rehabilitation, 31(12), 958-66, 2009.

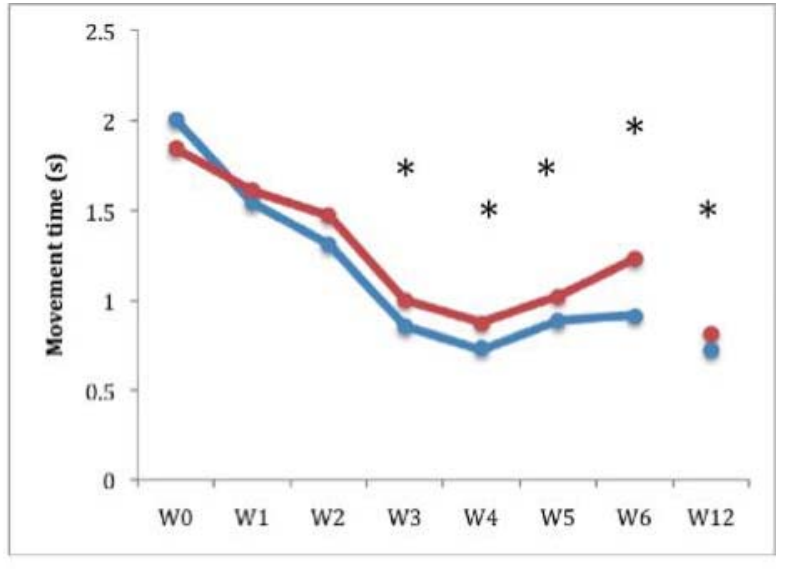

Fig. 1: Movement time (MT) in bimanual condition. The non-paretic hand is represented in blue and the paretic hand in red. The stars indicate a significant difference with MT at WO and W1.

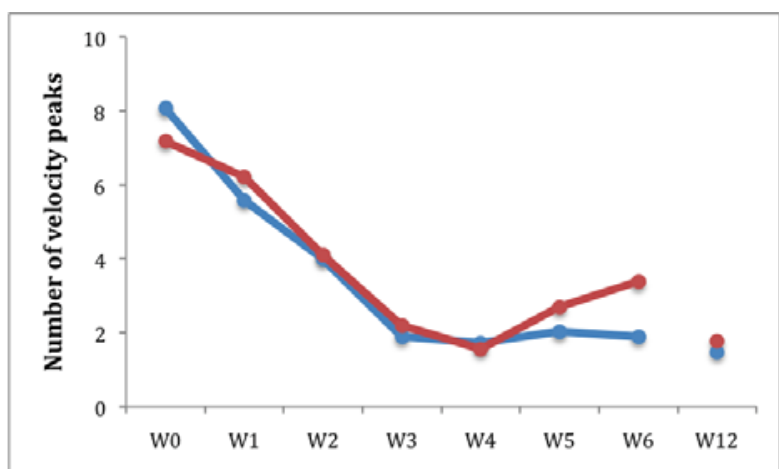

Fig. 2: Number of Peak Velocity (NVP) in bimanual condition. The non-paretic hand is represented in blue and the paretic hand in red.

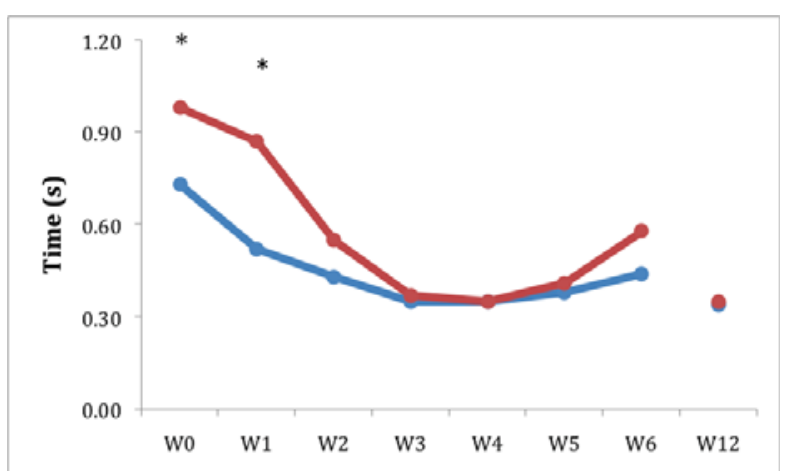

Fig. 3: Evolution of Time to Peak Velocity in bimanual condition. The non-paretic hand is represented in blue and the paretic hand in red. The stars indicate a significant difference between both hands. 